\title{
Review: Comparative Analysis of Different Techniques of DL-Frameworks
}

\author{
K. Krishna \\ PG student, SAL Institute of \\ technology \& Engineering \\ Research, Opp. Science city, \\ Sola Road, Ahmedabad-380060
}

\author{
B. Jigar \\ H.O.D. (EC/IC) SAL Institute of \\ technology \& Engineering \\ Research, Opp. Science city, \\ Sola Road, Ahmedabad-380060
}

\author{
M. Vyoma \\ Asst. Prof. SAL Institute of \\ technology \& Engineering \\ Research, Opp. Science city, \\ Sola Road, Ahmedabad-380060
}

\begin{abstract}
Deep learning is developed in 2006 and it is a part of machine learning and also for the artificial intelligence. Deep learning provides state of the arts solutions of various problems in areas like speech recognition and processing, neural language processing, image processing, computer vision etc. To prepare and simulate these kind of solution, there are many open source frameworks like Theano, TensorFlow, CNTK, caffe, Torchnet, Deep Learning $4 \mathrm{j}$ are available. Theano provides comparatively better hardware performance while TensorFlow provides better visualization by dataflow graphs. In this paper TensorFlow, Theano and CNTK will be compared on the basis of model Capability, Interface, Performance, Platform support, Speed, Distributed computing, Parallel execution. The best achievable goal of this work to display the best Deep Learning framework by implementing the neural network architecture for classifying images from several datasets. In above techniques some of the parameters of TensorFlow will give better performance than the others.
\end{abstract}

\section{Keywords}

Deep Learning, Datasets, NeuralNetwork, Performance Comparison, Theano, TensorFlow, CNTK.

\section{INTRODUCTION}

Deep learning is a new field in machine learning research. The key driving factor for these deep learning exercises to learn and study about human brain which replicates the process of human brain to identify and recognize data such as sounds, images and texts. The concept of deeplearning is derived from the study of artificial neural networks.

Because of so many recent developments in deep learning research, there are so many open source deep learning frameworks are available which include CNTK, TensorFlow and Theano etc. This paper will provide comparison of above frameworks based on their creator, development community its availability across various platforms, interface supports, supportsfor CUDA, OpenGL, OpenCL, Data parallelization, modeling capability andspeed [7][9].

\section{INTRODUCTION OF FRAMEWORKS}

\subsection{Tensor Flow}

Google Brain Team has developed TensorFlow. It is the second-generation machine learning systembased on DistBelief. It uses for speech recognition or image recognition and other deep learning.

The initial release was issued on November 9, 2015. The version 1.0.0 was released on February 11, 2017. Later on the stable release was issued on July 10, 2018. TensorFlow
Lite is design for Android development which supports Android Oreo (8) in May 2017.

Data flow graphs are used for computation in TensorFlow in which mathematical operations are represented by nodes and the data represented by edges. The communication among these edges are being done by multidimensional data arrays or tensors [2].

TensorFlow is basically written in a python language with $\mathrm{C} / \mathrm{C}++$ and provides interface for the same. It also supports CUDA library (cuDNN). TensorFlow is currently available for Linux, Windows, MacOS as well as for Android, iOS and Raspberry Pi.

TensorFlow has huge community over the world on GitHub and it is increasing. TensorFlowAPIs supports many languages including Keras, Go, Python, $\mathrm{C}++$, and Java. There is also a non-official (community-developed) bindings including C\#, Haskell, Julia, Ruby, Rust, and Scala [6].

TensorFlowarchitecture is given by as below.

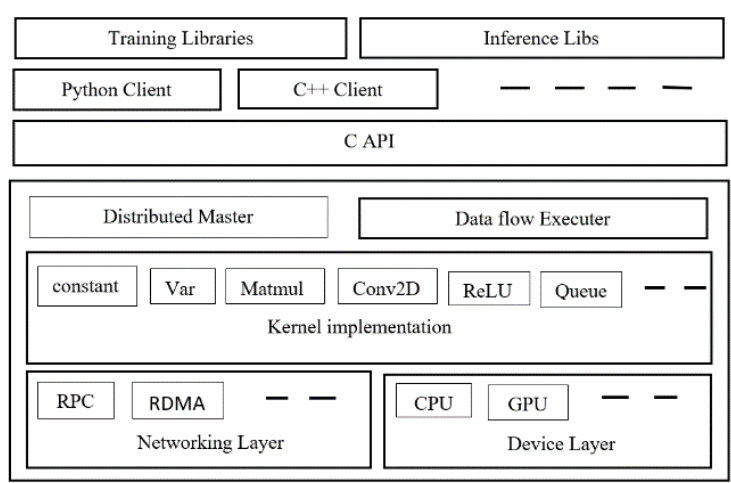

Figure-1General Architecture of TensorFlow [6]

It supports CNN, RNN and LSTM algorithms. TensorFlow is flexible because itcan run various models of the same modelsimultaneously. It provides better computation graph visualization and huge library support. It provides portability supports sowithout having any other hardware support it can be run on all platforms. In TensorFlow it supports pre-trained models which helps to carry out research and production processes faster. It has automatic differentiation capabilities. It also provides data parallelism in which parallel implementation of different models can be done simultaneously on multiple device.

It provides GPU supports for only NVIDIA GPUs. some updates were with no backward compatibility. There is some gap in documentation for beginning [8]. 


\subsection{CNTK}

Microsoft cognitive toolkit is known as CNTK is aopen source deep learning framework which is developed by Microsoft research and the first release was out on $25^{\text {th }}$ January, 2016. Later on stable release (2.5.1) was out on $17^{\text {th }}$ April, 2018.

CNTK is a deep learning framework that givesflexibility, efficiency, balancesand Performance in one package. The inspiration behind CNTK was Lego Bricks in which, each brick is very simple and performs a specific function but by combining many bricks an arbitrary objectcan be created [2].

To make edits and changes in framework CNTK model provides interface for Network definition language (NDL) and model editing language (MEL) with $\mathrm{C}++, \mathrm{C} \#$ and Python It supports 64-bit Linux, Windows operating system it also supports MacOS via Docker on roadmap.

The general architecture of CNTK is given as below.

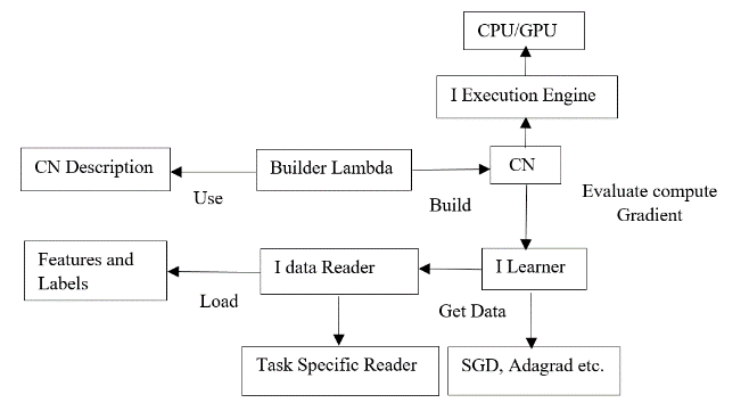

Figure-2 General Architecture of CNTK [9]

CNTK Modelling SupportsCPU and GPU with a focus on GPU Cluster. For GPU, it uses NVIDIA libraries, including cuDNN v5.

Currently CNTK interface are supported in Python (Keras), $\mathrm{C}++$, Command line, BrainScript and also in .NET on roadmap. It also provides OpenMP, Recurrentnets, Convolutional nets. Currently Open CL support is not available in CNTK.

CNTK has automatic numerical differentiation capabilities. By batching it's providing more efficient recurrent network training and static. Model execution is comparatively faster than other frameworks because it supports data parallelization by sharing of memory during execution planning.

\subsection{Theano}

Theano is developed at MILA Lab, university of Montreal. It is using NumPy's syntax to optimize and evaluate mathematical expression. Theano 1.0 was released on $28^{\text {th }}$ September, 2017 then on $15^{\text {th }}$ November, 2017 Theano 1.0.0 wasreleased.

Theano is basically written only in python language. It also supports CUDA Deep Neural Network library (cuDNN), OpenMP, OpenCL supports is in under process. Theano is cross platform framework and available for Linux, Windows, MacOS, CentOS 6 and Gentoo [10].

It supports CNN, RNN, RBM and DBNalgorithms. Itsupports Pre-trained model as well as Recurrent nets and Convolutions nets.

Some deep-libraries such as Keras, Lasagne and Blocks which are using theano for deep networks. This makes theano highly extensible. Theano can be run parallel on multiple devices by parallelexecutionvia multi node. Same as CNTK it has automatic numerical differentiation capability.

Theano cannot be deployed on multiple GPU so Large models can demand long compile times. While using on AWS it is quite difficult to use and the error messages details for debugging are not so helping [4].

\section{COMPARISION OF FRAMEWORKS}

There are so many deep learning modelingsoftware are currently available in market but in this paper, Author have seen major three frameworks which are TensorFlow, Microsoft Cognitive Toolkit also popularly known as CNTK and Theano.

For a better decision making of which framework should be used, the comparisons of above framework on variousparameters must be done. In this paper various parameters like platform support, language used to write that framework, the interface provided to edit or make changes in respected framework, supports for CUDA, OpenMP, OpenCL. Recurrent nets, Convolution nets, Pre-trained models and many more have been considered [1]

The detailed table of comparison is given by below table1.[11]

Table 1. Comparison between TensorFlow, CNTK and Theano.

\begin{tabular}{|c|c|c|c|}
\hline & TensorFlow & $\begin{array}{l}\text { Microsoft Cognitive Toolkit } \\
\text { (CNTK) }\end{array}$ & Theano \\
\hline Creator & Google Brain Team & Microsoft Research & University of Montreal \\
\hline Software license & Apache 2.0 & MIT License & BSD License \\
\hline Open Source & Open source & Open source & Open source \\
\hline Platform Supports & $\begin{array}{l}\text { Linux, macOS, Windows, } \\
\text { Android }\end{array}$ & $\begin{array}{l}\text { Windows, Linux (macOS via } \\
\text { Docker on roadmap) }\end{array}$ & Cross Platform \\
\hline Written In & $\mathrm{C}++$, Python, CUDA & $\mathrm{C}++$ & Python \\
\hline Interface & $\begin{array}{l}\text { Python (Keras), C/C++, Java, } \\
\text { Go, R, Julia }\end{array}$ & $\begin{array}{l}\text { Python (Keras), C++, } \\
\text { Command line, BrainScript, } \\
\text {.NET on roadmap }\end{array}$ & Python (Keras) \\
\hline OpenMP & Not supported & Supported & Supported \\
\hline
\end{tabular}




\begin{tabular}{|c|c|c|c|}
\hline OpenCL & $\begin{array}{l}\text { On roadmap but already with } \\
\text { SYCL support }\end{array}$ & No supported & Under Development \\
\hline CUDA & Supported & Supported & Supported \\
\hline $\begin{array}{l}\text { Parallel Execution (Multi } \\
\text { Mode) }\end{array}$ & Supported & Supported & Supported \\
\hline $\begin{array}{ll}\text { Automatic } & \text { Numerical } \\
\text { Differentiation } & \end{array}$ & Supported & Supported & Through Lasagne's model zoo \\
\hline Pre-trained Model Support & Supported & Supported & Supported \\
\hline Recurrent nets & Supported & Supported & Supported \\
\hline Convolution nets & Supported & Supported & Supported \\
\hline $\begin{array}{l}\text { Modeling } \\
\text { Capability }\end{array}$ & $\begin{array}{l}\text { RNNs, } \\
\text { CNNs, } \\
\text { RBMs, } \\
\text { DBNs, } \\
\text { LSTMs. }\end{array}$ & $\begin{array}{l}\text { CNNs, } \\
\text { RNNs, } \\
\text { LSTMs, } \\
\text { FNNs, } \\
\text { KNNs. }\end{array}$ & $\begin{array}{l}\text { RNNs, } \\
\text { CNNs, } \\
\text { RBM, } \\
\text { DBNs. }\end{array}$ \\
\hline Model Execution Speed & Fast & Faster & Fast \\
\hline
\end{tabular}

\section{CONCLUSION}

After doing detailed analysis on comparison table, there are several facts that should be considered while making decision regarding which framework should be choose.

All frameworks support distributed computing, If the modeling data is too big and required quite a big computation power (Use of multiple GPU), then user should pick TensorFlow or CNTK because currently Theano can be run on one GPU at a time.

For the users who wants to run framework using ARM architecture (Use of Mobile device) they can use TensorFlow because currently CNTK and Theano are not available on ARM architecture.

TensorFlow andCNTK are providing various interfaces for editing framework while theanoonly edited with Python (Keras) support.

TensorFlow and CNTK has built in Automatic numerical differentiation capability while in theano it can be done via third party support.

Theano has extensive libraries like Lasagne, Keras, Blockwhich can create models in lesser time. On other hand TensorFlow has unique and powerful tool named TensorBoard for debugging and visualization of created model.

Overall compare to other frameworks, TensorFlow is a widely used framework worldwide with huge libraryand worldwide active members with greater Community support for development and supports.

\section{REFERENCES}

[1] Dong-sheng GAO, Yan-rong ZHAO, Jing GAO and Hao WANG, "Comparison and Analysis of the OpenSourceFrameworks for Deep Learning”, 2016 International Conference on Mathematical, Computational and Statistical Sciences and Engineering (MCSSE 2016), ISBN: 978-1-60595-396-0,240,2016.

[2] Ali Shatnawi, Ghadeer Al-Bdour, Raffi Al-Qurran and Mahmoud Al-Ayyoub, “A Comparative Study of Open
Source Deep Learning Frameworks", 2018 9th International Conference on Information and 11Communication Systems (ICICS), IEEE,978-1-53864366-2/18,72,2018.

[3] Rub'en D. Fonnegra, Bryan Blair, Gloria M.D'iaz, "Performance Comparison of Deep LearningFrameworks in Image Classification Problems usingConvolutional and Recurrent Networks", IEEE, 978-1-5386-1060-2/17,2017.

[4] AniruddhaParvat, Jai Chavan, SiddheshKadam, Souradeep Dev, Vidhi Pathak, "A Survey ofDeep-learning Frameworks", International Conference on Inventive Systems and Control (ICISC-2017), IEEE, 978-1-50904715-4/17,1,2017.

[5] Shaohuai Shi, Qiang Wang, Pengfei Xu, Xiaowen Chu, "Benchmarking State-of-the-Art Deep Learning Software Tools", 2016 7th International Conference on Cloud Computing and Big Data, IEEE,978-1-5090-3555$7 / 16,99,2016$

[6] https://www.tensorflow.org for information about TensorFlow.

[7] https://developer.nvidia.com/deep-learning for information about CUDA support.

[8]https://docs.microsoft.com/en-us/cognitive-toolkit for information about CNTK.

[9] Alexey Kamenev, Microsoft Research "Deep Learning in Microsoft with CNTK”.

[10] http://deeplearning.net/software/theanofor information about theano.

[11] en.wikipedia.org for information about various deep learning framework. 